\title{
EP-154
}

\section{Early experience of ambulatory laparoscopic cholecystectomy in Thailand}

\author{
Nattawut KEERATIBHARAT*
}

Department of Surgery, Surnaree University of Technology, Nakhon Ratchasima, Thailand

Introduction: The benefits of laparoscopic cholecystectomy (LC) include faster recovery, earlier return to work and less postoperative pain when compared to open cholecystectomy. Ambulatory laparoscopic cholecystectomy is an established practice in the western country due to cost effectiveness and safety, but it is not well established in Thailand. The aims of our study are to demonstrate the applicability and safety of ambulatory laparoscopic cholecystectomy from our early experience.

Methods: Data was collected retrospectively for ambulatory laparoscopic cholecystectomy from January 1, 2018 to June 30, 2018. Patient demographic data, peri-operative data and post-operative data were collected and analyzed.

Results: Six patients were underwent ambulatory laparoscopic cholecystectomy in our hospital and all patient were discharged on the day of surgery. Patients satisfaction rate was 100\%. Mean operative time was 55 minutes and mean blood loss was $13.7 \mathrm{~mL}$. There were no unexpected hospitalization and prolong stay in our study.

Conclusions: Ambulatory LC can be performed safely in our early experience. Ambulatory LC in Thailand is still new method for patients and some physicians. The further study from our hospital may encourage ambulatory LC for standard method in gallstone disease in Thailand. 